Revue française de la traduction

\title{
Ergonomie et appropriation : un exemple d'évolution de l'utilisation des outils de traduction
}

\section{Caroline Champsaur et Michel Rochard}

\section{(2) OpenEdition}

\section{Journals}

Édition électronique

URL : http://journals.openedition.org/traduire/175

DOI : $10.4000 /$ traduire.175

ISSN : 2272-9992

Éditeur

Société française des traducteurs

\section{Édition imprimée}

Date de publication : 1 juin 2011

Pagination : 7-13

ISSN : 0395-773X

\section{Référence électronique}

Caroline Champsaur et Michel Rochard, «Ergonomie et appropriation : un exemple d'évolution de I'utilisation des outils de traduction », Traduire [En ligne], 224 | 2011, mis en ligne le 03 février 2014, consulté le 02 mai 2019. URL : http://journals.openedition.org/traduire/175 ; DOI : 10.4000/

traduire.175 


\section{Ergonomie et appropriation : un exemple d'évolution de l'utilisation des outils de traduction}

Caroline Champsaur et Michel Rochard

L'histoire récente (dix ans) de l'Unité des références et de la terminologie (URT), unité de documentation de la Division de la traduction de l'OCDE, est emblématique de l'ambivalence des traducteurs face à l'évolution de leur métier : un mélange de crainte devant la modernité (les outils vont tuer le métier) et de volonté de ne pas subir l'outil, mais de l'assujettir à leurs besoins professionnels.

Pour bien comprendre cette expérience concrète que nous illustrerons par des exemples portant sur différents outils, il faut préalablement sortir d'un schéma dans lequel la traduction est un ensemble d'opérations organisées de façon linéaire. Si tel était le cas, I'URT de l'OCDE serait vraisemblablement restée un simple prestataire de services informatisés apportés aux traducteurs au fil des étapes balisées de leur travail. En revanche, si l'on se place dans le cadre d'une activité assimilable à une enquête non linéaire(1), on comprend mieux l'évolution des rapports d'un groupe de quelques dizaines de traducteurs avec les nouveaux outils de "l'industrie de la langue".

\section{Terminologie : de la boîte à chaussures à l'outil collaboratif}

La terminologie est révélatrice de l'ambiguïté des rapports entretenus par les traducteurs avec les outils informatisés de la traduction. Boîte à chaussures de fiches " bristol ", petit répertoire ou cahier, boîte à fiches en métal ou en plastique, les traducteurs ayant blanchi sous le harnais ont connu ou pratiqué ces formules. Quant au contenu de ces trésors, il pouvait tout aussi bien rester caché, être distillé goutte à goutte par leur propriétaire, ou mis plus largement à la disposition d'un collectif. Dans les organisations internationales, la terminologie a eu tendance à constituer le premier outil informatique en dehors du traitement de texte. À l'OCDE, il était géré centralement sous DOS dans le cadre de notre unité, I'URT. Les bases de données ainsi

(1) Rochard (1999). 
constituées réunissaient les travaux terminologiques des "élus ", car seuls les réviseurs étaient à l'époque habilités à produire de la terminologie publiable. L'URT apportait alors un service de terminologie, sous forme de réponses validées aux questions des traducteurs.

Une première tentative de base de travail contributive plus large a été mise en place vers 1995, mais elle ne correspondait plus aux besoins de terminologie raisonnée exprimés par plusieurs traducteurs. En effet, ces derniers, sans être réviseurs, avaient des antécédents ou exprimaient des besoins terminologiques plus poussés. Vinrent alors les suites bureautiques modernes et celle adoptée à l'OCDE a permis de construire des bases de données répondant assez bien à ces besoins. L'individualisme de la boîte à chaussures n'a pas pour autant aussitôt disparu car chaque base présentait des champs différents et n'était accessible qu'à la personne qui l'administrait.

Plusieurs facteurs ont contribué à l'appropriation de ces bases de données par la plupart des traducteurs du service. La pluridisciplinarité des textes a poussé d'une part les traducteurs à s'intéresser aux travaux terminologiques de leurs collègues et d'autre part les administrateurs de base à rendre leur contenu plus accessible. L'URT y a aussi contribué en assurant les migrations successives des bases de données au fil des versions des logiciels, ce qui a permis une certaine standardisation de la présentation et de la structure des bases et leur réunion sous un lecteur commun de notre serveur. De ce fait, de nouvelles vocations terminologiques sont nées, la gestion individuelle des bases est devenue partagée et, l'évolution technologique aidant, l'idée de réunir tous ces travaux dans le cadre d'une même application informatique a pu prendre corps. Enfin, avec l'arrivée de nouvelles générations de traducteurs plus portées à dialoguer avec les auteurs, l'idée d'associer les auteurs à nos travaux s'est imposée. Les outils de Web2 et les portails collaboratifs sont entre-temps apparus, venant conforter cette démarche. Nous sommes donc, par des voies détournées, revenus à une normalisation et une centralisation des travaux terminologiques, sans pour autant priver chaque traducteur spécialiste de son rôle moteur dans ses domaines de prédilection.

Dans ce processus, l'URT est passée d'un rôle d'apporteur de réponses terminologiques à une position plus stratégique. C'est en effet l'URT qui rédige actuellement l'appel d'offres pour acquérir l'application de portail qui répondra aux besoins de l'ensemble de l'OCDE (services auteurs, service des publications, traducteurs et interprètes) et qui sera maître d'œuvre d'un "Termium de l'OCDE ".

Même si l'individualisme des démarches terminologiques n'a pas complètement disparu, on peut dire qu'il y a eu appropriation de l'outil terminologique par les traducteurs au service du collectif, ce qui était impensable il y a 15 ans(2).

(2) Voir aussi Rochard (2008). 


\section{Les informations documentaires}

De tout temps, les traducteurs ont sollicité les services de l'URT pour connaître des intitulés d'organes officiels, de projets, de réunions ou de travaux. À l'URT travaillaient alors des " documentalistes " qui faisaient les recherches demandées et consignaient précieusement les informations trouvées sur des fiches en papier. À l'époque, l'information était rare. Elle se devait d'être conservée par des personnes dignes de confiance (les documentalistes en l'occurrence) et communiquée aux traducteurs uniquement si ces derniers en faisaient la demande.

Le passage au support informatique n'a rien changé : l'information était consignée dans des bases non consultables par les traducteurs et ces derniers devaient venir en personne solliciter une information auprès d'un documentaliste qui effectuait une recherche dans la base au moyen d'une syntaxe alambiquée.

C'est le passage à un logiciel Windows ayant une interface web pour les utilisateurs et ne nécessitant aucune installation sur le poste client qui a radicalement changé le rapport à l'information : les traducteurs n'ont plus besoin de se déplacer, ils peuvent consulter euxmêmes la base et obtenir le renseignement recherché.

Dans un premier temps, l'accueil de ce nouvel outil a pourtant été mitigé : les traducteurs avaient l'impression qu'ils étaient négligés et considéraient cette obligation de recours à un outil informatique comme une perte de qualité dans le service rendu. De même, le renseignement trouvé sur cet outil paraissait moins fiable et moins complet que celui que communiquait une personne humaine dont on pouvait mesurer le temps de recherche. Le sentiment prédominant était donc la méfiance !

Peu à peu, pourtant, les traducteurs ont compris l'intérêt de faire des recherches dans cette base, validée par des humains identifiés, plutôt que dans une base contenant certes tous les documents de l'Organisation, mais comportant de nombreuses erreurs et incohérences ou, pire, sur l'Internet où la source était encore moins sûre. Les traducteurs sont alors progressivement devenus des acteurs : ils signalent les erreurs à l'équipe de l'URT, demandent l'ajout de précisions ou d'entrées nouvelles. Au fil du temps, cet outil est devenu, aux yeux des traducteurs, un de "leurs " outils et non plus un outil de l'URT.

\section{Outils de reprise de passages traduits}

L'arrivée d'un logiciel de mémoire de traduction a suscité autant d'espoirs que de déceptions. L'outil ne venait pas répondre à un véritable besoin préexistant (les passages repris n'étaient pas si nombreux avant les traitements de texte). Toutefois, les traducteurs mesuraient pleine- 
ment l'intérêt de retrouver des passages " déjà traduits " et, encore mieux, " déjà traduits par eux-mêmes ".

Cependant, la complexité d'utilisation de ce logiciel a découragé de nombreux traducteurs et bientôt, c'est de nouveau l'URT qui a servi de médium pour trouver les passages repris. Là encore, réaction méfiante : d'où venaient les passages repris trouvés par l'URT ? Les traducteurs étaient-ils obligés de reprendre les traductions préexistantes? Ces passages repris allaient-ils être comptabilisés dans leur "production " en nombre de pages traduites ?

Dans un deuxième temps cependant, certains traducteurs sont venus trouver I'URT pour " commander " des comparaisons de leur texte à traduire avec un corpus de textes défini par eux. De "receveurs passifs ", ces traducteurs sont donc devenus " donneurs d'ordre". Parallèlement à cette modification des rapports, les traducteurs ont été nettement plus satisfaits des résultats obtenus... même lorsque ceux-ci ne donnaient pas satisfaction! En effet, si la comparaison ne donnait pas ou très peu de passages repris, le traducteur pouvait se lancer dans sa traduction sans risque de retraduire des choses traduites antérieurement. Si la comparaison s'avérait fructueuse, le traducteur s'affranchissait ainsi de traduire des passages de son texte. Dans les deux cas, l'outil était utile.

L'arrivée d'un logiciel de recherche permettant de créer ses propres corpus ("DtSearch ॥) a été mieux accueillie : un réviseur du service féru de nouvelles technologies a construit un large corpus à destination de l'ensemble du service. Chaque traducteur était alors libre d'utiliser ces textes pré-indexés, mais aussi d'indexer lui-même d'autres textes. Certes, les résultats obtenus ne sont pas très rapides (il faut copier chaque passage recherché dans une fenêtre spécifique, lancer la recherche parmi les textes indexés, sélectionner un texte dans une liste de résultats puis copier-coller la traduction ainsi trouvée). Néanmoins, les traducteurs travaillent à présent en parfaite autonomie, s'affranchissant totalement des services de I'URT. Ce point a paru positif à l'ensemble des traducteurs. Cependant, il a son revers : les corpus ainsi créés ne sont pas partagés, avec le risque de création parallèle de corpus identiques, les méthodes de travail ont tendance à varier et, en fin de compte, on peut déplorer un manque d'harmonisation des sources.

\section{Le portail fédérateur}

Un nouvel outil, lancé au printemps 2010, devrait aider à harmoniser les pratiques des traducteurs et favoriser les échanges, tout en laissant chacun libre de ses méthodes de travail : le portail. Un portail est un site où chaque utilisateur est automatiquement identifié, ce qui permet :

- au gestionnaire d'afficher des informations ciblées pour cet utilisateur, 
- à l'utilisateur de personnaliser le portail et de l'enrichir de ses propres sources pour qu'il réponde à ses propres besoins,

- aux utilisateurs d'échanger des commentaires sous forme d'un "blog " ou d'une autre technologie " Web 2.0 " comparable.

Le portail de la traduction a été très bien accueilli par les traducteurs du service qui ont immédiatement apprécié son caractère de " sésame " (il donne immédiatement accès - par simple clic - à une très large palette d'outils utilisés précédemment). Le portail a donc été perçu dans un premier temps comme une façon de simplifier l'utilisation des autres outils.

Dans un second temps, les traducteurs ont exploré les différentes sources offertes (références préparées par l'URT, glossaires, sites documentaires, liens vers des sources pertinentes pour la traduction) et ont apprécié la richesse des références offertes.

La fonction d'échanges (blogs, commentaires sur les textes à traduire) n'a pas encore été adoptée : les traducteurs préfèrent toujours l'échange direct avec un collègue, plutôt que d'utiliser un outil laissant une trace écrite visible par tous. Cependant, avec l'augmentation du nombre de traducteurs travaillant hors site (traducteurs externes, télétravailleurs...), cette fonctionnalité sera peut-être plus appréciée dans les années qui viennent.

Le succès du portail tient à plusieurs aspects :

- sa facilité d'utilisation (pas de mot de passe, simple clic sur les liens...),

- sa spécificité : c'est un portail pour les traducteurs et non pas un site d'informations générales,

- sa richesse : plus l'outil a des chances d'être réellement utile, moins le traducteur hésitera à l'utiliser,

- sa personnalisation : chaque traducteur peut adapter cet outil à ses besoins et choisir soit de partager ses informations avec l'ensemble des traducteurs (comme dans le cas de la base documentaire en ligne), soit d'ajouter des informations à des fins personnelles uniquement.

Enfin, le portail a réussi à créer de nouveaux liens :

- entre l'URT qui collecte puis publie des informations et les traducteurs qui réagissent et participent au choix des informations diffusées,

- entre les traducteurs qui partagent un même espace dédié renforçant leur identité spécifique mais qui restent libres de garder une autonomie dans le choix et le scénario d'utilisation des différents outils. 


\section{Conséquences organisationnelles de l'appropriation des outils}

Nous avons d'emblée évoqué l'attitude ambivalente des traducteurs face aux outils : les outils vont tuer le métier, donc je m'approprie les outils pour assurer la pérennité du métier ! Toute stratégie d'introduction de nouveaux outils doit donc tenir compte du paradoxe suivant : c'est la peur de la modernité qui pousse à être moderne.

\section{Une stratégie souple}

Les responsables de services de traduction doivent éviter de définir leur stratégie de modernisation comme une suite linéaire de versions informatiques du poste du traducteur : adoption du traitement de texte (Version 1.0), ajout des bases de données terminologiques (Version 2.0), ajout de l'indexation (Version 3.0), ajout de la TAO (Version 4.0), jusqu'à l'introduction de la Version n.0, à savoir la traduction automatique(3). En effet, un service de traduction est un ensemble présentant à la fois une pyramide des âges et une pyramide des compétences informatiques qui ne se recoupent pas nécessairement. Une stratégie linéaire va se heurter à cette réalité et risque d'aboutir à un service à plusieurs vitesses.

La stratégie de modernisation doit être souple. Elle doit d'abord reposer sur une veille technologique pragmatique. Cette veille ne doit pas être menée in vitro par des "technos ", mais être interactive : un outil ne doit pas être mis en service sans être testé par des traducteurs de profils différents. Ensuite, la stratégie doit anticiper l'appropriation par les traducteurs de l'outil qui va vraisemblablement changer de statut dans le travail de l'unité chargée de la modernisation : du service clé en main, à l'entretien de l'outil, au conseil à l'utilisation, en passant par la remontée des critiques des traducteurs vers le fournisseur externe. La stratégie doit être collaborative.

\section{Une structure intégrée}

L'un des éventuels travers des services de traduction dotés d'une unité de type URT est que cette unité s'autonomise totalement vis-à-vis du reste du service. Elle devient alors un État dans l'État et finit par imposer " la modernité " comme une fin en soi(4). Cette dernière est alors subie de façon autoritaire par les traducteurs. C'est contraire à l'évolution même de l'informatique depuis l'avènement de l'Internet. C'est également contraire à l'évolution des formations de traducteurs qui intègrent de plus en plus la dimension de l'industrie de la langue dans leurs cursus. La présence d'un ensemble de documentalistes, de terminologues, de traducteurs spécialistes de l'industrie de la langue, et de spécialistes de l'informatique linguistique dans le cadre d'une telle unité est un gage de diversité et d'ouverture, en particulier vers les traducteurs.

(3) On pourrait y ajouter la dictée analogique, la dictée numérique, la reconnaissance vocale, etc.

(4) C'est ce qui s'est produit avec les services informatiques de grandes entreprises ou organisations. 


\section{Pour conclure}

L'ergonomie de la traduction est un enjeu stratégique pour l'efficience des services de traduction. C'est un lourd convoi dont l'industrie de la langue est le moteur, mais ce moteur a besoin de ces relais de transmission que sont in fine les traducteurs.

Caroline CHAMPSAUR est docteur en linguistique théorique et formelle et chef de l'Unité de références et terminologie au sein de la Division de la traduction de l'OCDE.

Michel ROCHARD est diplômé de traduction de l'Université de Mayence à Germersheim, docteur en traductologie de I'Université de Paris 3 (ESIT), réviseur à la Division de la traduction de I'OCDE et chargé de cours à I'UFR EILA (Paris 7) et à l'ESIT (Paris 3).

\section{Références}

DEWEY John, 1933, How we think, Boston, D.C. Heath \& Co

DEWEY John, 1938, Logic: The theory of inquiry. In J.A. Boydston (Ed.), John Dewey: The later works, 1925-1953. (Vol 12: 1938). Carbondale, IL, Southern Illinois University Press, 1986

ROCHARD M., 1992, Le traducteur face à la terminologie : consommateur ou acteur?, Commission des Communautés européennes, Revue Terminologies et Traduction, $n^{\circ} 2 / 3$

ROCHARD M., 1999, Traduction professionnelle et traduction pédagogique : le lien de l'enquête, in Lavault Elisabeth et Lafon M., Les Cahiers de l'Institut des langues et cultures de l'Europe (ILCE), Université Stendhal - Grenoble 3

ROCHARD M., 2008, Le traducteur-terminologue et l'expert, rencontre hypertextuelle, in Société française des traducteurs, Traduire $\mathrm{n}^{\circ} 217$ 